\author{
Research Article
}

\title{
Invadopodia Formation is a Critical Step in Cancer Cell Invasion: The Effect of Passage Number on Invadopodia Formation in MDA-MB-231 Breast Cancer Cell Line
}

\author{
Hamad Ali Hamad ${ }^{1}$, Cheah Yoke Kqueen ${ }^{1}$, and Nur Fariesha MD Hashim ${ }^{1, *}$ \\ 1 Department of Biomedical Science, Faculty of Medicine and Health Sciences, Universiti Putra Malaysia, 43400 Serdang, Selangor Darul Ehsan, Malaysia \\ https://doi.org/10.28916/lsmb.2.3.2018.20 \\ Received 28 July 2018, Revisions received 23 November 2018, Accepted 23 November 2018, Available online 25 December 2018
}

\begin{abstract}
High invasive cancer cells are thought to recruit specialised actin-rich protrusions for invasion in metastasis process. These protrusions are termed invadopodia. To study invadopodia formation, one of the first challenges faced by researchers has been to optimise the cell line passage number in order to be used for the invadopodia assay. Therefore, this study aims to investigate the effects of the passage number on invadopodia formation in MDA-MB-231 breast cancer cell line. Invadopodia assay was used to achieve the aim of the study. The results provided evidence that invadopodia formation is affected by the high passage number. The cells were also tested with dimethyloxalylglycine (DMOG) a hypoxic mimicking agent which is known to be an invadopodia inducer, the results showed that the cells in low passage number (P7) treated with DMOG increase the cells forming invadopodia, while the cells with high passage number (P35) showed that DMOG fails to stimulate the cells to form invadopodia. Furthermore, the cells with high passage number after passage 15 are starting to lose the ability to degrade the gelatin. In conclusion, this study suggests that only cells with a low passage number, less than passage 15 should be used in the study of invadopodia formation to obtain the results in the search for molecular targets and signaling at invadopodia.
\end{abstract}

Keywords: Invadopodia, passage number, DMOG, $M D A-M B-231$ cell line

\subsection{Introduction}

Breast carcinoma cells need to invade the surrounding extracellular matrix (ECM) to achieve metastasis successfully. Metastasis is a multistep process in which the cancer cells spread from primary breast tumor site to a secondary site such as bone or brain (Paz et al., 2014). The majority of cancer mortalities are primarily due to metastasis (Xie et al., 2017), therefore investigation of the mechanism of cancer cells to invade the ECM during metastatic cascade is critical in cancer study (Bowden et al., 1999).

In recent years, actin-rich subcellular protrusions have been shown to be critical for ECM degradation. Accordingly, these protrusions are termed invadopodia and are formed in the ventral of highly invasive cancer cells. Invadopodia consists of actin core surrounded by several essential proteins for ECM degradation, namely; matrix metalloproteinases (MMPs), Abl kinase signalling, cortactin, fascin-1, LPA1 receptor and EGFR (Harper et al., 2018; Paz et al., 2014; García et al., 2016; Smith-Pearson et al., 2010; Beghein et al., 2018). Invadopodia are reported to be formed by various types of malignant cells, including the MDA-MB-231 breast cancer cell line. In a study reported by Hashim et al., (2013), the invasiveness of MDA-MB-231 breast cancer cells increases due to increased expression of the hypoxia-inducible factor-1 $\alpha$ (HIF$1 \alpha$ ) under hypoxic environment. Furthermore, invadopodia formation also found increased in MDA-MB-231 cells when treated with a cellpermeable prolyl-4-hydroxylase inhibitor dimethyloxalylglycine (DMOG) (Salvi et al., 2017; Hashim et al., 2013). HIF-1 $\alpha$ promotes invadopodia formation in hypoxia conditions.

Studies showed that the overexpression of HIF-1 $\alpha$ increases the number of cells forming invadopodia as well as increased the degradation of gelatin due to the molecular component activation of invadopodia such as MT1-MMP, MMP2, Arp2/3, $\beta$-PIX, and MMP-9 (Egeblad and Werb, 2002; Hashim et al., 2013; Gould and Courtneidge, 2014). The effect of hypoxia on breast cancer cell invasion in MDAMB-231 cell line is dramatically higher than MDA-MB-435 cell line. Moreover, MMPs activation cascades are essential in the hypoxic MDAAMB-231 cell. The previous report has shown that there are two proteins significantly increased in hypoxia and important for invadopodia to degrade ECM, those proteins are MT1-MMP and MMP-2 (Castro-Castro et al., 2016; Poincloux et al., 2009). In addition, when designing an experiment, it is important to optimise the cell line passage number especially in the study on invadopodia formation. Passage number, the number of periods a cell culture has been subcultured. Many previous and more recent studies on invadopodia formation in various types of cancer cell lines have been carried out on the signaling and regulation of invadopodia. However, none of these studies identified the effect of the passage number on invadopodia formation (Gurski et al., 2012; Choi et al., 2013; Harper et al., 2018; Ren et al., 2018).

\footnotetext{
* Correspondence: Department of Biomedical Science, Faculty of Medicine and Health Sciences, Universiti Putra Malaysia, 43400 Serdang, Selangor Darul Ehsan, Malaysia

e-Mail: nurfariesha@upm.edu.my
}

(C) 2018 by the Author(s). Life Sciences, Medicine and Biomedicine (ISSN: 2600-7207) Published by Biome Journals. Wholly owned by Biome Scientia Sdn Bhd., previously by WWH Networks Sdn Bhd. Attribution-ShareAlike 4.0 International (CC BY-SA 4.0). This open access article is distributed based on the terms and conditions of the Creative Commons Attribution license https://creativecommons.org/licenses/by-sa/4.0/ 
In this study, invadopodia assay was used to investigate the effect of the passage number on invadopodia formation of MDA-MB231 cells. Thus, the present study aims to use DMOG as inducer to activate HIF-1 $\alpha$ that is known to increase invadopodia formation in low passage number. Though there is no evidence on MDA-MB-231 cells if can form invadopodia in high passage number and if can response to DMOG to form invadopodia in high passage number as well. The findings of the study will provide the researcher with a mini guideline to study invadopodia in MDA-MB-231 cell line.

\section{Materials and methods}

\section{Cell culture and maintenance}

MDA-MB-231 cell line was cultured in DMEM (Dulbecco modified Eagle medium) high glucose medium containing $10 \%$ FBS (fetal bovine serum), and $1 \%$ pen/strep (penicillin and streptomycin). The MDA-MB-231 breast cancer cell line was purchased from American Type Cell Culture Inc (ATCC Manassas, VA, USA). The cells were thawed and collected to falcon tube containing $5 \mathrm{~mL}$ media then centrifuged for $5 \mathrm{~min}, 250 \mathrm{RCF}$ at $4{ }^{\circ} \mathrm{C}$, after that, the supernatant was discarded and added $2 \mathrm{~mL}$ DMEM media to the pellet, then the cells are re-suspended and transferred to T25 flask contain $3 \mathrm{~mL}$ DMEM media then incubated at $37^{\circ} \mathrm{C}$ with humidified air of $5 \% \mathrm{CO}_{2}$ until $80 \%$ confluence to avoid post-confluent growth. The cells were then washed twice with PBS (phosphate buffer saline) after discarding the medium. Next, $1 \mathrm{~mL}$ of $0.25 \%$ trypsin was added to the flask and incubated for $5 \mathrm{~min}$ at $37^{\circ} \mathrm{C}$ until the cells de-attached. The cells were next harvested into falcon tube and centrifuged for $5 \mathrm{~min}, 250 \mathrm{RCF}$ at $4^{\circ} \mathrm{C}$. After that, re-suspend the cells with $4 \mathrm{~mL}$ media then the cells were counted then seeded into a six-well plate each plate contain $5 \times 10^{4}$ cells/ $\mathrm{mL}$ for invadopodia experiment. The remaining cells were subcultured for next invadopodia experiment. The cells were observed daily under the microscope (Leica Microsystems, Wetzlar, Germany) and regularly examined for the presence of Mycoplasma contamination by Hoechst stain.

\section{$2.2 \quad$ Gelatin coated coverslip preparation}

Oregon Green ${ }^{\circledR} 488$ Conjugate from Porcine Skin (Invitrogen) was used to coat round glass coverslip (12 mm) before seeding with MDA-MB-231 cells. The protocol was adapted from (Enderling et al., 2008; Harun et al., 2018). The working concentration of gelatin was $0.2 \mathrm{mg} / \mathrm{mL}$ from stock $1 \mathrm{mg} / \mathrm{mL}$, and the working concentration of gelatin was achieved by dilution with PBS containing $2 \%$ sucrose. Each coverslip was then placed onto parafilm with $40 \mu \mathrm{L}$ of $0.2 \mathrm{mg} / \mathrm{ml}$ gelatine for $10 \mathrm{~min}$ where the coverslips were then transferred using forceps onto a new parafilm with $100 \mu \mathrm{L}$ of $0.5 \%$ glutaraldehyde solution. Following 15 min of incubation in a dark humidified environment, the coverslips were transferred into a 24-well plate with the gelatin-coated side facing up followed by washing 3 times with PBS. Then 1 mL of sodium $5 \mathrm{mg} / \mathrm{ml}$ borohydride was added to each well for $3 \mathrm{~min}$, shaking every minute to avoid air bubbles. This was followed by discarding the sodium borohydride and washing gently 3 times with PBS and 3 times $70 \%$ ethanol. Finally, the ethanol was aspirated off, and the coverslips were left to dry on the bench for $2 \mathrm{~min}$ in complete darkness. The gelatin-coated coverslips could be stored at $4{ }^{\circ} \mathrm{C}$ for a maximum of one week or used directly in the Invadopodia assay. For invadopodia assay, the gelatin-coated coverslips were quenched with DMEM medium for $1 \mathrm{hr}$ in a tissue culture prior seeding cell.

\section{3}

\section{Invadopodia assay}

The low passage or high passage number of MDA-MB-231 cells were seeded into a 6-well plate, with each well-containing $5 \times 10^{4}$ cells/ $\mathrm{mL}$ and incubated overnight. This was followed by $6 \mathrm{hr}$ incubation in the case of treatment with $0.5 \mathrm{mM}$ DMOG. Next, the gelatincoated coverslips were quenched with DMEM medium for $1 \mathrm{hr}$ in a tissue culture incubator where the cells were then re-trypsinised and reseeded $\left(2 \times 10^{4}\right.$ cells $\left./ \mathrm{mL}\right)$ onto a gelatin-coated coverslip for precisely $3 \mathrm{hrs}$. The incubation of the cells on the gelatin-coated coverslips had been optimised to avoid cells moving to other areas of the gelatine after degrading the matrix (Hashim et al., 2013). Following 3 hrs, the medium was discarded, and the coverslips were gently washed one time with PBS where the cells were then fixed with $4 \%$ paraformaldehyde for 20 min. The coverslips were permeabilised with $0.2 \%$ Triton X-100 in PBS for 5 min after being washed gently 3 times with PBS. The cells were next stained with Rhodamine phalloidin (Invitrogen) diluted in $3 \%$ BSA in PBS for $1 \mathrm{hr}$ in a dark humidified environment followed by staining the cells with Hoechst 33342 (Invitrogen) for $10 \mathrm{~min}$ to label the nucleus and to observe any signs of mycoplasma infection (Chen, 1977). This was followed by mounting the gelatin-coated-coverslips onto ProLong Gold antifade (Invitrogen). The slides were then ready to be observed for invadopodia formation under a fluorescent microscope. The cells were scored for the presence of underlying invadopodia and the mean percentage of cells with invadopodia formation was calculated. The sample size for each experiment was 100 cells from three independent experiments.

\subsection{Statistical analysis}

Around 15-20 fields were randomly captured from each coverslip to quantify invadopodia formation in the cells. The values calculated from the three independent experiments were compared by conducting one-way ANOVA test, using GraphPad Prism 6. All groups were compared with low passage number (Passage 6). The results showed the control is passage number 6, which is the best passage number after thawing the cells from passage number 4 . Moreover, $P<0.05$ is considered statistically significant. Error bars represent the standard deviation (SD) of mean.

\section{Results and Discussion}

Breast carcinoma cells need to invade the surrounding extracellular matrix (ECM) to achieve metastasis successfully (Paz et al., 2014). Thus, invadopodia formation has been known to degrade ECM and allow the malignant cells to migrate to the blood vessels (García et al., 2016). Passage number is one of the first cell culture practices taught in cell culture laboratories. It is important for many reasons, the most obvious being to create an adequate number of cells to obtain enough cells to perform the experiments (Crisostomo et al., 2006).

Many of the previous reports focus on the effect of passage number in primary cells because their lifetime is limited. Also, the primary cells might change their behavior and function (Chennazhy and Krishnan, 2005; Calles et al., 2006). However, limited studies have been found on cancer cells. Thus, the present study aims to use invadopodia assay to investigate the effects of passage number on invadopodia formation in MDA-MB-231 cell line. The results provide evidence that the cells with high passage number (Passage 35) of MDA-MB-231 cells are affected invadopodia formation thereby making it unable to accomplish gelatin degradation as a black spot (Figure 1A). While the 
cells with low passage number (Passage7) form invadopodia as a black spot on gelatin (Figure 1B). Furthermore, passage number 6, 7 and 10 are forming invadopodia normally with no statistical significance (Figure $2 \mathrm{~A}$ ). However, from passage number 15 onwards the number of cells that form invadopodia are statistically declined until passage number 35; whereby invadopodia formation almost disappears (Figure 2A). In addition, to observe whether invadopodia inducer (DMOG) can stimulate invadopodia formation in a low passage number (Passage 7) and high passage number (Passage 35), MDA-MB-231 cells in passage number 7 and passage number 35 were treated and incubated for 6 hrs with $0.5 \mathrm{mM}$ DMOG. DMOG a chemical compound well known to mimic hypoxia and induce invadopodia in the MDA-MB-231 cell line (Hashim et al., 2013).

The results showed the inducer DMOG in passage 7 has successfully increased the number of cells to form invadopodia to $59 \%$ compared with $34 \%$ in grow (passage 7) as control (Figure 2B), whereas, in passage 35 the inducer DMOG fail to stimulate the cells to form invadopodia (Figure 2B). Therefore, the cells with high passage number could miss their signaling and molecular components of invadopodia formation that are essential to degrade ECM (Meirson and Gil-Henn, 2018; Jacob and Prekeris, 2015; Maziveyi et al., 2018). A study on LNCaP human prostate cancer cell line considered passage 60 as the highest passage number and passage 25 as the lowest passage, whereas PI3K/Akt pathway influenced by passage number (Esquenet et al., 1997) However, invadopodia formation in MDA-MB-231 cells shows influence after passage 15 . Thus, the data could be used as a mini guideline to study invadopodia formation, especially in the highly invasive MDA-MB-231 breast cancer cell line. To validate these results, other invasive cancer lines should be tested for invadopodia formation in different passages to ensure that researchers can refer to the correct passage number(s) for optimal invadopodia formation.

F. actin

A

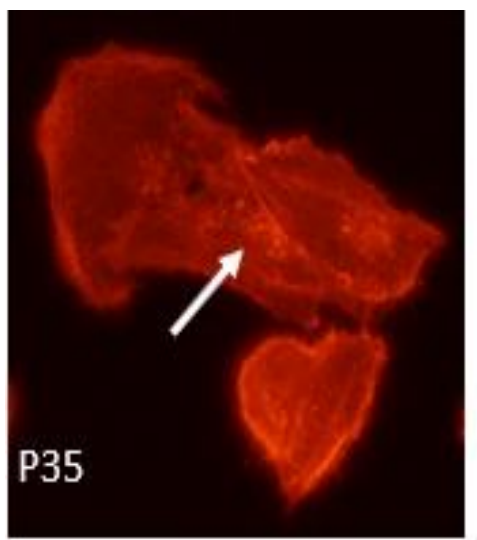

B

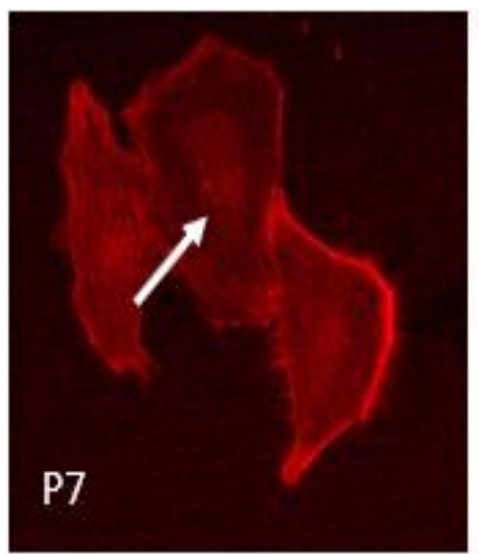

Gelatin and nucleus
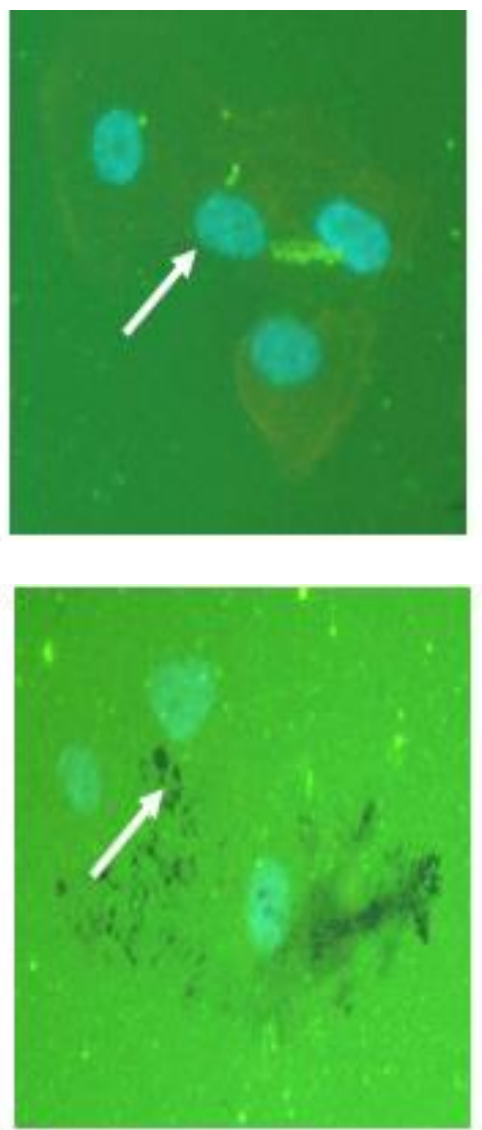

Merge
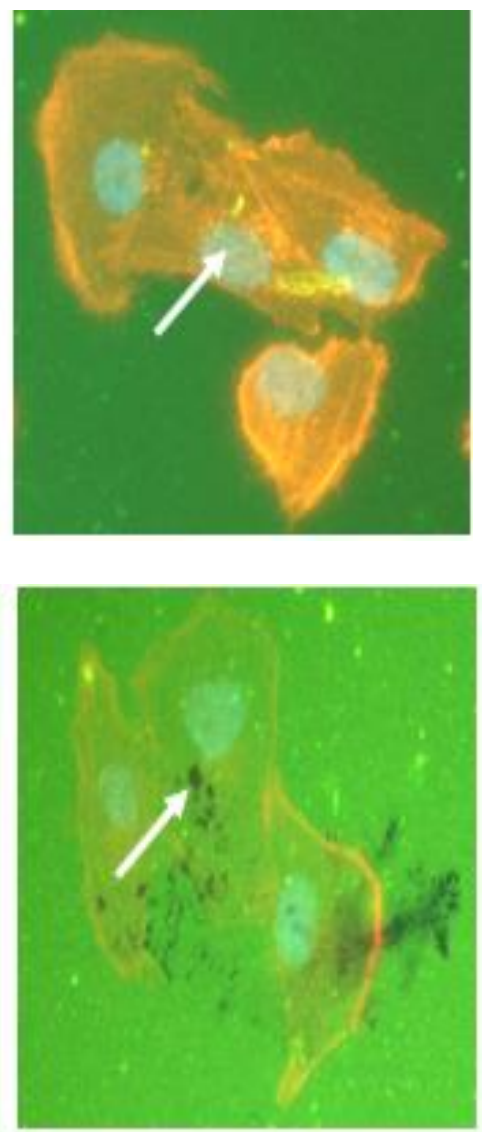

Figure 1: The effect of passage number on invadopodia formation in low passage number (P7) and high passage number (P35). (A) Cells with high passage number (P35) show no gelatin degradation as noted in the arrow in green color images. (B) Cells with low passage number (P7) show gelatin degradation in green color images as a black spot and the red image shows cytoskeleton. $\mathrm{P}=\mathrm{Passage}$ 

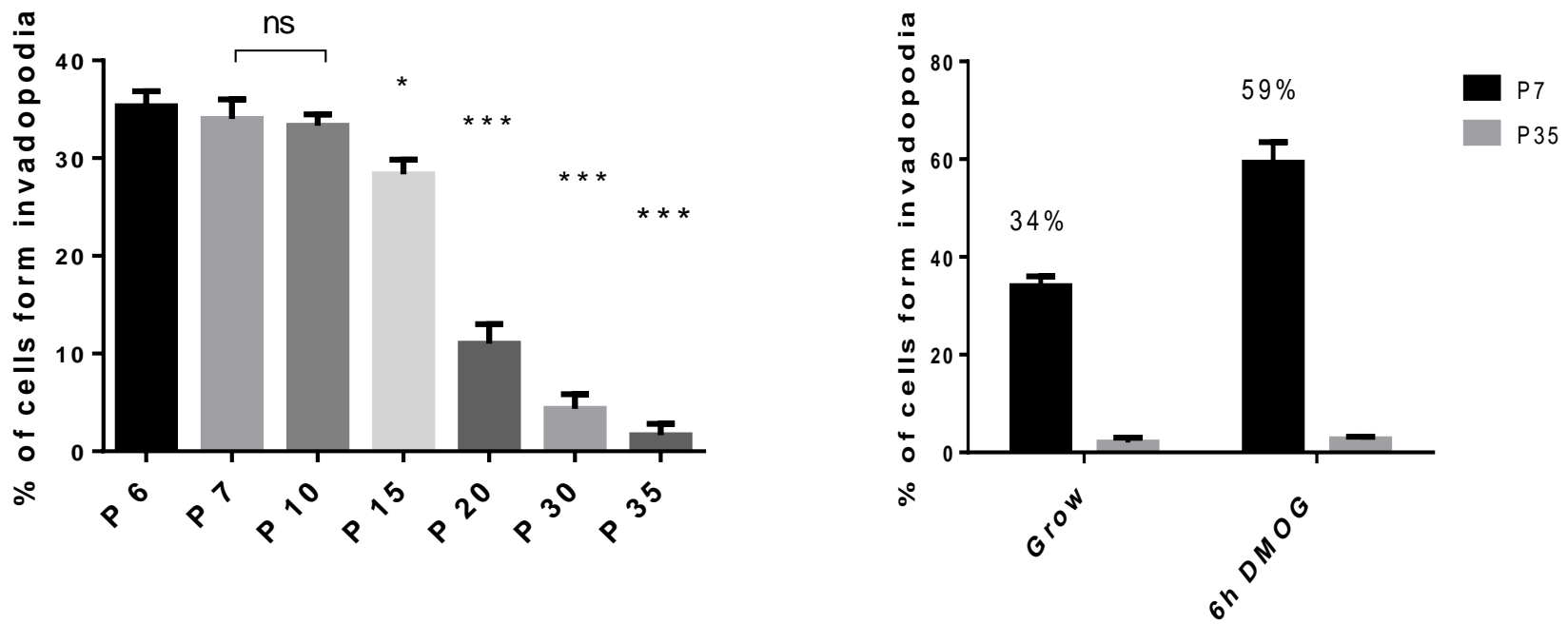

Figure 2: Evaluation of the effect of passage number on invadopodia formation in MDA-MB-231 cells. (A) The effect of invadopodia formation on a variety of passage numbers. (B) The effect of passage number on invadopodia formation in low passage number (P7) and high passage number (P35) in cells treated with DMOG as invadopodia inducer. Statistical analysis was applied to reveal the significance when the measurements compared to passage 6 in (A) and passage 7 in (B) as a control by GraphPad Prism 6 (one-way ANOVA). Data presented as (Mean \pm standard deviation). $* \mathrm{P}<0.05$, *** $\mathrm{P}<0.0005$, significant. $(\mathrm{n}=3$, sample size 100 cells each experiment), $(\mathrm{P}=\mathrm{Passage}$, $\mathrm{ns}=$ not significant).

\section{Conclusion}

Invadopodia formation is a primary step in metastasis. To target invadopodia and its molecular components, future researches should consider the passage number. The findings of the study suggested that the best accurate passage number to be used in invadopodia formation study must be less than passage 15 . The present study could be used as a mini guideline to study invadopodia formation, especially in highly invasive MDA-MB-231 breast cancer cell line.

\subsection{Conflict of Interest}

The authors declare no conflict of interest.

\section{Acknowledgements}

The research work in this study is supported by Geran Putra IPM; project number GP-IPM/2016/7470300. Universiti Putra Malaysia, 43400 Serdang, Selangor. The authors thank Cell Signaling Unit for providing facilities throughout this research.

\subsection{References}

Beghein, E., Devriese, D., Van Hoey, E., \& Gettemans, J. (2018). Cortactin and fascin-1 regulate extracellular vesicle release by controlling endosomal trafficking or invadopodia formation and function. Scientific reports, 8, 15606.

Bowden, E. T., Barth, M., Thomas, D., Glazer, R. I., \& Mueller, S. C. (1999). An invasion-related complex of cortactin, paxillin and PKC $\mu$ associates with invadopodia at sites of extracellular matrix degradation. Oncogene, 18, 4440-4449.

Calles, K., Svensson, I., Lindskog, E., \& Häggström, L. (2006). Effects of conditioned medium factors and passage number on Sf9 cell physiology and productivity. Biotechnology progress, 22, 394-400.

Castro-Castro, A., Marchesin, V., Monteiro, P., Lodillinsky, C., Rossé, C., \& Chavrier, P. (2016). Cellular and molecular mechanisms of MT1MMP-dependent cancer cell invasion. Annual review of cell and developmental biology, 32, 555-576.

Chellini, L., Caprara, V., Spadaro, F., Sestito, R., Bagnato, A., \& Rosanò, L. (2018). Regulation of extracellular matrix degradation and metastatic spread by IQGAP1 through endothelin-1 receptor signaling in ovarian cancer. Matrix Biology. doi.org/10.1016/j.matbio.2018.10.005

Chen, T. R. (1977). In situ detection of mycoplasma contamination in cell cultures by fluorescent Hoechst 33258 stain. Experimental cell research, 104, 255-262.

Chennazhy, K. P., \& Krishnan, L. K. (2005). Effect of passage number and matrix characteristics on differentiation of endothelial cells cultured for tissue engineering. Biomaterials, 26, 5658-5667.

Choi, J. A., \& Lim, I. K. (2013). TIS21/BTG2 inhibits invadopodia formation by downregulating reactive oxygen species level in MDA-MB231 cells. Journal of cancer research and clinical oncology, 139, 1657-1665.

Clark, E. S., Whigham, A. S., Yarbrough, W. G., \& Weaver, A. M. (2007). Cortactin is an essential regulator of matrix metalloproteinase secretion and extracellular matrix degradation in invadopodia. Cancer research, 67, 4227-4235.

Crisostomo, P. R., Wang, M., Wairiuko, G. M., Morrell, E. D., Terrell, A. M., Seshadri, P., Nam, U. H., \& Meldrum, D. R. (2006). High passage number of stem cells adversely affects stem cell activation and myocardial protection. Shock, 26, 575-580.

Egeblad, M., \& Werb, Z. (2002). New functions for the matrix metalloproteinases in cancer progression. Nature Reviews Cancer, 2, 161-174.

Enderling, H., Alexander, N. R., Clark, E. S., Branch, K. M., Estrada, L., Crooke, C., Jourquin, J., Lobdell. N., Zaman, M, H., Guelcher, S, A., Anderson, A. R., \& Weaver, A. M. (2008). Dependence of invadopodia function on collagen fiber spacing and cross-linking: computational modeling and experimental evidence. Biophysical Journal, 95, 2203-2218. 
Esquenet, M., Swinnen, J. V., Heyns, W., \& Verhoeven, G. (1997). LNCaP prostatic adenocarcinoma cells derived from low and high passage numbers display divergent responses not only to androgens but also to retinoids. The Journal of steroid biochemistry and molecular biology, 62, 391-399.

García, E., Ragazzini, C., Yu, X., Cuesta-García, E., De La Serna, J. B., Zech, T., Zech, T., Sarrió, D., \& Antón, I. M. (2016). WIP and WICH/WIRE co-ordinately control invadopodium formation and maturation in human breast cancer cell invasion. Scientific reports, 6 , 23590.

Gould, C. M., \& Courtneidge, S. A. (2014). Regulation of invadopodia by the tumor microenvironment. Cell adhesion \& migration, 8, 226235.

Gurski, L. A., Xu, X., Labrada, L. N., Nguyen, N. T., Xiao, L., van Golen, K. L., Jia, X., \& Farach-Carson, M. C. (2012). Hyaluronan (HA) interacting proteins RHAMM and hyaluronidase impact prostate cancer cell behavior and invadopodia formation in 3D HA-based hydrogels. PLoS One, doi: 10.1371/journal.pone.0050075.

Harper, K., Lavoie, R. R., Charbonneau, M., Brochu-Gaudreau, K., \& Dubois, C. M. (2018). The Hypoxic Tumor Microenvironment Promotes Invadopodia Formation and Metastasis through LPA1 Receptor and EGFR Cooperation. Molecular Cancer Research, 16, 1601-1613.

Harun, S. N. A., Israf, D. A., Tham, C. L., Lam, K. W., Cheema, M. S., \& Md Hashim, N. F. (2018). The Molecular Targets and Anti-Invasive Effects of 2, 6-bis-(4-hydroxyl-3methoxybenzylidine) cyclohexanone or BHMC in MDA-MB-231 Human Breast Cancer Cells. Molecules, 23, 865. doi: 10.3390/molecules23040865.

Hashim, N. F. M., Nicholas, N. S., Dart, A. E., Kiriakidis, S., Paleolog, E., \& Wells, C. M. (2013). Hypoxia-induced invadopodia formation: a role for $\beta$-PIX. Open biology, 3, 120159 doi: 10.1098/rsob.120159

Jacob, A., \& Prekeris, R. (2015). The regulation of MMP targeting to invadopodia during cancer metastasis. Frontiers in cell and developmental biology, 3,4

Maziveyi, M., Dong, S., Baranwal, S., \& Alahari, S. K. (2018). Nischarin regulates focal adhesion and Invadopodia formation in breast cancer cells. Molecular cancer, 17, 21. doi: 10.1186/s12943-018-0764-6.

Meirson, T., \& Gil-Henn, H. (2018). Targeting invadopodia for blocking breast cancer metastasis. Drug Resistance Updates, $39,1-17$.

Paz, H., Pathak, N., \& Yang, J. (2014). Invading one step at a time: the role of invadopodia in tumor metastasis. Oncogene, 33, $4193-4202$.

Poincloux, R., Lizárraga, F., \& Chavrier, P. (2009). Matrix invasion by tumour cells: a focus on MT1-MMP trafficking to invadopodia. $J$ Cell Sci, 122, 3015-3024.

Ren, X. L., Qiao, Y. D., Li, J. Y., Li, X. M., Zhang, D., Zhang, X. J., ... \& Liao, W. T. (2018). Cortactin recruits FMNL2 to promote actin polymerization and endosome motility in invadopodia formation. Cancer letters, 419, 245-256.

Salvi, A., \& Thanabalu, T. (2017). Expression of N-WASP is regulated by HiF1 $\alpha$ through the hypoxia response element in the N-WASP promoter. Biochemistry and biophysics reports, 9, 13-21.

Smith-Pearson, P. S., Greuber, E. K., Yogalingam, G., \& Pendergast, A. M. (2010). Abl kinases are required for invadopodia formation and chemokine-induced invasion. Journal of Biological Chemistry, jbc-M110. doi: 10.1074/jbc.M110.147330

Xie, H. Y., Shao, Z. M., \& Li, D. Q. (2017). Tumor microenvironment: driving forces and potential therapeutic targets for breast cancer metastasis. Chinese journal of cancer, 36, 36. doi: 10.1186/s40880-017-0202-y 